\title{
DESAFIOS DE LA EDUCACION EN EL FUTURO DE LA UNIVERSIDAD
}

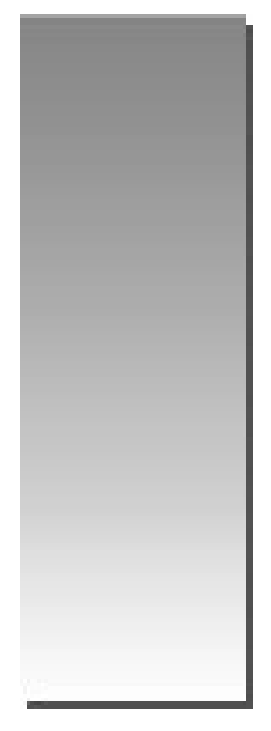

Ebor Fairlie Frisancho*

E-mail: efairlie@unmsm.edu.pe

\section{RESUMEN}

El presente artículo de investigación reúne algunas opiniones sobre lo que podemos esperar de la educación en un futuro quizás no tan lejano, al menos en América Latina y España. Las opiniones son de investigadores de universidades, quienes analizan los objetivos, desafíos, inversión, cultura y estrategias.

El papel del Banco Mundial es imponderable para la mejora de los estudios y se presentan diversas alternativas para tomar en cuenta y entrar al reto de mejorar la educación en los centros de enseñanza superior en concordancia con los estándares de calidad establecidos por los países desarrollados.

Palabras clave: Educación del Futuro, tuning, Informe PISA, Banco Mundial, investigación de la educación.

\section{ABSTRACT}

The present article gathers some opinions about that we can expect of the education in a perhaps not so distant future, at least in Latin America and Spain. The opinions are of researches of universities, who they analyze the objectives, challenges, investment, culture and strategies.

The role of the World Bank is imponderable for the improvement of the studies and diverse alternatives are presented to take into account and to enter to the challenge to improve the education in the higher education institutions in agreement with the standards of quality established by the countries developed

Key words: Education of the future, tuning, PISA report, World Bank, investigation of the education.

* Estudios de Doctorado en Administración. Magíster en Administración. Licenciado en Administración de Empresas. Profesor auxiliar e investigador de la Unidad de Investigaciones de la Facultad de Ciencias Administrativas. Asesor de la Oficina de Calidad Académica de la Facultad de Ciencias Administrativas (UNMSM). 


\section{INTRODUCCIÓN}

Se habla mucho de lo que puede suceder en el futuro, de cómo serán las futuras universidades, qué incidencia tendrán en la educación, y si algún factor externo, desafío global o inversión económica, entre otros, pueden influenciar a los centros de enseñanza superior.

Diversas instituciones, entre ellas las Naciones Unidas y el Banco Mundial, han desarrollado y auspiciado estudios sobre la educación en diversas universidades latinoamericanas, entre ellas nuestra universidad. Estas instituciones presentan informes anuales para ver el avance relativo, acorde con las políticas educativas planteadas.

Los resultados siempre han sido alentadores desde el punto de vista de avance y desarrollo pero muy alejados de la realidad de otras economías desarrolladas. Diversos informes como el Informe de Programa para la Evaluación Internacional de Alumnos, PISA 2007, ubican al Perú en penúltimo lugar de rendimiento a pesar de tener más de 83 universidades en todo el país, ello debido a que no existe una política acorde a un plan o a una meta fijada para un año específico.

\section{MARCO TEÓRICO}

La investigación con respecto al Estado del Futuro 2007, es un informe recientemente elaborado con respecto a la situación y desafíos globales del futuro. Sus autores son Jerome Glenn y Theodore Gordon quienes han desarrollado toda una investigación en base al análisis de cuestionarios y entrevistas para obtener resultados sobre el nivel educativo y compararlos con los años anteriores.

Así tenemos que esta investigación revisa los desafíos globales (que suman quince); el índice del Estado del futuro; educación y aprendizaje al 2030 y problemas emergentes sobre seguridad ambiental. Los autores indican que este tipo de investigación resulta de especial importancia debido a que:

"El objetivo de las investigaciones futuristas es explorar, crear y evaluar de forma sistemática tanto los futuros posibles como los deseables, en busca de la mejora en el proceso de toma de decisiones. La toma de decisiones se ve afectada por la globalización; de ahí que la investigación futurista a nivel global será necesaria para mantenerse informado sobre las decisiones tomadas por particulares, grupos e instituciones"1.
En todo país existen problemas y oportunidades y este informe puede dar algunas luces sobre cómo afrontar exitosamente el futuro que, a entender propio, se acerca rápidamente. En la actualidad muchos de los problemas que enfrentamos en diversos ámbitos (desde el familiar hasta el nacional), son un conglomerado de varios ámbitos y etapas, así tenemos que las soluciones tienen que abarcar otras naciones, otras instituciones y otras disciplinas para que éstas sean perdurables en el tiempo.

A opinión de diversos tratadistas, los investigadores académicos tienen menos ámbitos de aplicación en el tiempo, dado que el ámbito de estudio se reduce considerablemente, cosa que no sucede con el futuro ya que se consideran posibilidades futuras dado que no es una ciencia (los estudios Delphi sirven perfectamente como un método futurista).

Entre los quince desafíos propuestos se encuentran algunos muy interesantes como:

- Desarrollo sustentable.

- Población y recursos.

- Capacidad de decisión.

- Conservación de la energía.

- Ciencia y tecnología.

- Entre otros.

Pero ¿por qué la educación no se encuentra dentro de estos desafíos globales? ¿acaso es un desafío o se encuentra inmerso en cada uno de ellos a través de las investigaciones que se deben de realizar previamente? ¿No contribuyen las universidades o los estudiosos a determinar cada uno de estos desafíos?

\section{LA EDUCACIÓN EN EL FUTURO}

De acuerdo a la investigación del Dr. Juan Carlos Tudesco presentada en su artículo "Los pilares de la educación del futuro", el dinamismo de los cambios en la sociedad se reflejan en la educación, la que necesariamente se ve obligada a afrontar nuevos retos.

Diversos cambios como un aumento significativo de la desigualdad social, evolución de la tecnología, exclusión social en el ciclo productivo, entre otros, afectan la educación y es necesario pensar en un nuevo contexto para afrontar nuevos retos. 
Se postula que se necesitan dos retos: el primero surge desde el desarrollo permanente y continuo, y está específicamente orientado a priorizar el proceso de aprendizaje para continuar aprendiendo. Este autor indica una desunión de los centros de enseñanza, las carreras profesionales que ofrecen y su localidad, y por ende no promueven programas masivos de intercambio entre las comunidades, barrios, fronteras, etc.

“... la autonomía y la individualización tampoco son incompatibles con la definición de objetivos comunes, de estándares comunes en términos de aprendizaje, cuya responsabilidad le cabe al Estado central tanto en su definición como en su regulación".

El segundo reto es la consecución de una identidad pero con un orden social preestablecido, en donde se pueda vivir cohesionadamente. En otras palabras la producción de conocimientos sigue incrementándose a un nivel geométrico y todos nosotros podemos acceder a un nivel de información casi infinito gracias a muchos recursos tecnológicamente creados para tal fin. Si esto sucede, entonces significa que cada hora, minuto o segundo llega nueva información para todo ámbito y nos obliga a estar informados; por lo tanto la obsolescencia será cada vez más rápida. Como dice el autor:

"En estas condiciones y para decirlo rápidamente, la educación ya no podrá estar dirigida a la transmisión de conocimientos y de informaciones sino a desarrollar la capacidad de producirlos y de utilizarlos."

Cabe destacar que en un artículo anterior disertamos sobre la veracidad y la calidad de la información, dado que en Internet se puede colocar lo que se desee y todo está al alcance del usuario que, ante esta gran masa de conocimientos, se ve imposibilitado de diferenciar entre información veraz y de calidad de aquella emitida por las personas como simple opinión.

\section{FUTURO SOSTENIBLE}

De acuerdo a la investigación de Amparo Vilches y Daniel Gil, profesores de la Universidad de Valencia, nuestra sociedad presenta una serie de elementos perjudiciales que se llaman elementos contaminantes como la contaminación espacial, lumínica, visual y acústica con la incidencia del agotamiento continuo de los recursos. Las me- didas educativas por sí solas no pueden erigirse en solución; han de apoyarse en un correcto conocimiento de la situación lo que implica serios estudios científicos, tecnológicos y la cuidadosa planificación de las acciones que se derivan; es decir, realizar complejas acciones en el campo científico-tecnológico.

Los mismos autores proponen lo siguiente:

- Formar instituciones que promuevan un nuevo orden mundial basado en la cooperación, solidaridad y defensa del medio ambiente, y evitar la imposición de valores e intereses particulares que resulten nocivos para la población actual o para las generaciones futuras.

- Impulsar una educación solidaria-superadora de comportamientos orientados por valores e intereses particulares- que contribuya a una correcta percepción de la situación del mundo, prepare para la toma de decisiones fundamentadas e impulse comportamientos dirigidos al logro de un desarrollo culturalmente plural y físicamente sostenible

- Dirigir los esfuerzos de la investigación e innovación hacia el logro de tecnologías favorecedoras de un desarrollo sostenible. incluyendo desde la búsqueda de nuevas fuentes de energía hasta el incremento de la eficacia en la obtención de alimentos, pasando por la prevención de enfermedades y catástrofes o la disminución y tratamiento de residuos, con el debido control social para evitar aplicaciones precipitadas.

Nuevamente la educación es una de las propuestas de estos investigadores porque es la base del conocimiento.

\section{NUEVOS DESAFIOS}

En la revista de investigación Universidades de la Universidad Autónoma del Estado de México, los investigadores Gilda Walkman y Luis Gurovich indican en su artículo "Tendencias, desafíos y oportunidades de la educación superior en el siglo XXI" la presencia de cinco mecanismos que están generando un cambio en la educación superior, los que están siendo adoptados por diversos países desarrollados y de América Latina. Estos mecanismos son:

- Multiplicidad de aplicaciones de la tecnología en la educación superior, como son las aulas virtuales y la educación a distancia. 
- Movilidad real y virtual de estudiantes y profesores, donde no existen ni fronteras ni idiomas.

- Educación permanente dada la cantidad exorbitante de información que día a día se incrementa; así tenemos, a la vez, información nueva e información obsoleta.

- Acercamiento al mundo laboral ya que no solo se requiere del conocimiento de la información sino de la experiencia obtenida en el mundo laboral, formación de equipos de trabajo, prácticas académicas, entre otros.

- Cambios en los roles de profesores y alumnos, ya que los primeros dejan de ser solo transmisores de conocimiento para asumir un rol de conducción de los alumnos.

Entonces los nuevos desafíos se convierten en la posible solución de problemas y dificultades como:

- La globalización que puede fomentar la desigualdad tanto al interior del país como a nivel internacional.

- El mercado de la educación no descansa solo en las propuestas de entidades privadas, sino que el Estado tiene que apoyar a las carreras de demanda social y vincular al sector productivo con la educación.

- La tecnología es la pista de despegue de nuevas formas de educación obligatoria y en donde el acceso rápido a la información es fundamental para el trabajo académico.

- El uso de redes determina la volatilidad de la investigación y eso implica la pérdida de lealtad institucional, generándose así nuevos criterios.

En resumen concluyen que la universidad, al tiempo que da respuesta a los desafíos que plantea la sociedad, no debe abandonar ni reducir su misión principal de investigadora y su compromiso con los alumnos.

\section{DIVERSAS CULTURAS, DIVERSA EDUCACION}

El Dr. Guillermo Williamson en su artículo "Acción afirmativa en educación superior: dilemas y decisiones en tiempos multiculturales de globalización”, postula que una educación de equidad para todos implica también oportunidades equitativas de acceso, permanencia y egreso en la educación superior universitaria. Esto es la Acción Afirmativa que no es otra cosa que:
“... la equidad en contextos de desigualdad creciente frente a las oportunidades, de diferenciación y de exclusión social. Las características de la globalización y de la multiculturalidad hacen que la sociedad civil, los movimientos sociales, el Estado e incluso la empresa privada busquen mecanismos institucionales, al menos de mitigación de esa situación educativa y social injusta".

Este artículo resume una serie de consecuencias, siendo las principales:

- La generación de nuevos problemas sociales (como pobreza, drogas, hacinamiento, enfermedades mentales, conflictos sociales y étnicos, inseguridad sobre el presente y el futuro, subculturas urbanas) asociados a una desigualdad creciente de la población, entre y al interior de los países

- La reorganización del trabajo que ha generado una división planetaria y al interior de las sociedades, entre un grupo que cuenta con la opción del trabajo que potencia sus capacidades humanas y una enorme masa de seres humanos sin acceso al empleo o producción o que si lo tiene, lo hace en condiciones de explotación, inseguridad y precariedad.

- La generación de mayores cambios ambientales provocados por el ser humano, que afectan la reproducción y seguridad de las diversas formas de vida, poniendo en riesgo las condiciones necesarias y convenientes para su feliz existencia y la reproducción de las futuras generaciones.

- Una reorganización a nivel mundial, regional, nacional y local de las estructuras y procesos de poder, fortaleciendo contradictoriamente la concentración económica y en consecuencia el poder político e institucional, con reacciones y resistencias -desde pacíficas a violentas- generadas desde la diversidad cultural e ideológica y las identidades locales.

\section{PROCESOS CURRICULARES DE LA EDUCACION}

En la revista Educación y Educadores, los profesores de la Universidad de Magdalena de España han propuesto el artículo "La educación superior frente a las tendencias sociales del contexto", donde explican que las instituciones educativas tienen que ajustarse a las tendencias internacionales pero conservando el desarrollo 
social de las comunidades. Para ello es necesario proveer de una formación básica, genérica y profesional sólida, que le permita al estudiante y egresado participar de manera autónoma, responsable y eficaz en la vida social y cultural. Además es necesario reforzar la fundamentación ética y la búsqueda de una conciliación entre desarrollo y equidad en los ciudadanos; elevar los niveles de exigencia y calidad en todos los procesos de formación universitaria, y crear y/o atender a indicadores de calidad de los procesos educativos planteados, a nivel nacional e internacional.

Una posible tendencia es el Proyecto Tuning que trata de afinar y relacionar áreas temáticas (empresariales, ciencias de la educación, geología, historia, matemáticas, física y química), mediante las definiciones aceptadas en común por resultados profesionales y de aprendizaje. Además busca desarrollar perfiles profesionales como resultado del aprendizaje y competencias deseables en términos de competencias genéricas y relativas, y facilitar la transparencia en las estructuras educativas e impulsar la innovación. El Proyecto Tuning plantea la creación de redes europeas capaces de presentar ejemplos de prácticas de innovación y calidad; desarrollar e intercambiar información relativa al desarrollo de los currículos en las áreas seleccionadas; crear una estructura curricular modelo expresada por puntos de referencia para cada área y generar puentes entre esta red de universidades y otras entidades apropiadas y calificadas, para producir convergencia en las áreas de las disciplinas seleccionadas.

Otra propuesta es el modelo de competencias que se basa en una propuesta hecha por Maldonado (2003) en la que la currícula tiene que reflejar competencias técnicas, metodológicas, sociales, participativas y de fundamentación básicas (habilidades, aptitudes y cualidades). El enfoque curricular integral basado en competencias tiene implicaciones en el proyecto educativo de la institución, en tanto que depende del tipo de ser humano que se quiere formar y del tipo de sociedad en la cual se quiere impactar.

Como conclusión podríamos decir que el currículo, como proceso de investigación, permite examinar e indagar de manera permanente y sistemática cada una de las acciones que se realizan durante el proceso de modernización curricular. Así tenemos que las reformas curriculares planteadas a nivel mundial favorecerán los procesos de internacionalización del currículo y la consolidación de redes académicas para el mejoramiento de la calidad, donde el papel del docente en uno de los pilares centrales de la construcción y desarrollo de estos procesos de modernización curricular.

\section{INCIDENCIA EN LA EDUCACION}

El Consejo de Educación de Adultos de América Latina organizó en Ecuador, durante el año 2006, un Taller de Incidencia en el que se analizó la incidencia política como herramienta eficaz de la sociedad civil para la implementación de políticas públicas, desde el punto de vista de la educación.

Se plantean diversas premisas. La más importante de ellas es que lo político debe incidir en la educación, lo que exige abandonar el enfoque sectorial y adoptar un enfoque multisectorial porque el desarrollo educativo de la familia, la comunidad y el país atraviesa a todos los ministerios y a todas las políticas. El propósito de la incidencia es avanzar en la concreción del derecho a la educación y en los cambios necesarios para lograrlo, por lo que no cabe apostar únicamente a una política educativa: es imprescindible incidir sobre el quehacer educativo y su entorno. Entonces encontramos que el cambio educativo -desde una perspectiva transformadora y popular- requiere de información, participación, organización y movilización en la base. (Ver Cuadro $\mathrm{N}^{\mathrm{0}} 1$ ).

A manera de conclusión podemos decir que en el Consejo de Educación de Adultos de América Latina se propuso:

- Superar la fragmentación, dispersión y hasta competencia entre la multiplicidad de actores que bregan por abrirse y sostener su propio espacio, desarrollando su propia agenda, todo ello agravado en el marco de un "campo educativo" crecientemente atomizado en niveles, ámbitos territoriales, tipos, modalidades de gestión, etc. La cultura de la fragmentación, el protagonismo y el corporativismo niega el sentido común de "la unión hace la fuerza" y corre en sentido contrario de cualquier empeño serio por incidir políticamente en educación o en cualquier otro campo.

- Romper con la dependencia técnica y financiera de las agencias internacionales que quita poder, resta autonomía, criticidad y capacidad para reconocer y analizar los problemas del viejo modelo de cooperación internacional 
para el desarrollo, que claramente y cada vez más forma parte del problema.

- Revisar los esquemas blanco/negro y la dualización Estado-gobierno/sociedad civil que ve al primero como el malo y a la segunda como la buena. Ni el Estado-gobierno es siempre antipopular, antidemocrático y corrupto, ni la sociedad civil es la heroína desinteresada de las buenas causas. También el gobierno es una entidad heterogénea y contradictoria que actúa bajo fuertes presiones internas y externas, y sus caras concretas varían mucho entre países así como en diversos escenarios y coyunturas. Gobiernos progresistas, embarcados en proyectos de justicia social, soberanía y dignidad, son potenciales aliados en la lucha contra los grandes poderes de las empresas multinacionales y de los organismos financieros internacionales que comandan el mundo globalizado y el rumbo de cada uno de nuestros países, y que vienen mostrando creciente interés en la educación no como derecho humano ni como motor de desarrollo sino como terreno de control y de negocio.

\section{OPINIÓN DEL BANCO MUNDIAL}

Entre el 6 y 7 de diciembre del 2007 se desarrolló el Primer Foro Nacional de Educación Superior con el tema de "Carreras estratégicas del futuro para el otorgamiento de becas nacionales". En ese evento el Banco Mundial presentó varias de las razones por las que hay que invertir en educación por ser un instrumento de desarrollo económico y social, las cuales son:

- Ayudar a los países para reducir la pobreza y mejorar sus niveles de calidad de vida.

- Apoyar el crecimiento sostenible y los avances tecnológicos.

- Apoyar la educación como inversión económica (en países desarrollados se destina del $50 \%$ al $60 \%$ de su riqueza nacional a la educación).

- Diseminar el conocimiento de los graduados a las nuevas generaciones.

Algunas premisas fueron indicadas:

- La educación mejora el trato a nivel de muchos sectores como por ejemplo el empoderamiento de la mujer y la agricultura.
- La desigualdad económica refleja una desigualdad educativa.

- La educación es una buena inversión, pero la inversión por parte de las personas no es la óptima.

- El mejor desempeño se observa por lo general en los países asiáticos en donde se da un alto valor social a la educación y tiene coherencia con el diseño de políticas orientadas a ésta.

\section{CONCLUSIONES}

1. El nivel de educación en los países indica un cierto nivel económico: mientras más brecha económica exista, habrá mucho mayor desigualdad en la educación.

2. El último informe del Programa para la Evaluación Internacional de Alumnos (PISA) de la Organización para la Cooperación y el Desarrollo Económico (OCDE), indica que nuestro país se encuentra en penúltimo lugar en el rendimiento de alumnos en cuatro áreas, colocando a Finlandia (que se ha superado) en primer lugar.

3. Esto es contradictorio ya que de acuerdo a la UNESCO 2006 estamos en un nivel intermedio en centros de enseñanza de educación superior, cosa que no se refleja en el punto anterior.

4. Las tendencias de crecimiento del empleo favorecen la mano de obra altamente calificada, por lo que la única forma de alcanzar la especialización es brindando una mejor educación a nivel de Post Grado.

5. El trinomio Estado-empresa-universidad debe estar presente en todo proceso de mejora de la educación.

6. Las economías asiáticas han tenido el éxito esperado porque han manejado políticas estables que se han mantenido permanente conforme pasan los gobernantes, cosa contraria a nuestro país.

7. El Estado debe brindar mucho mayor apoyo a la educación, en especial a las entidades del Estado, pero si se mantienen los recortes presupuestarios y el poco dinero que se recibe es destinado a fines distintos a los educativos, seguiremos con una gran inestabilidad que a la larga no nos favorece. 
NOTA

1. Situación del Futuro 2007.

\section{BIBLIOGRAFÍA}

GARCÍA ARETIO, L. (2001). La Educación a Distancia. De la Teoría a la Práctica. Capítulo 5 (Profesores / formadores y tutores). Madrid, Ariel Educación.

GISBERT, M. (2000). Pupitre-net: plataforma virtual para la integración de los recursos educativos sobre Internet. En PÉREZ PÉREZ, R. (Coord.). Redes multimedia y diseños virtuales. Oviedo, Servicio de Publicaciones de la Universidad de Oviedo.

GRACIA DE MILLÁN, SONNIA (2005). Curso Experto Universitario en Administración de la Educación. Ensayo inédito. OEI-UNED.

MARTÍNEZ MIGUÉLEZ, M. (2002). Un nuevo paradigma para la educación. Parte de la conferencia presentada en el Congreso Internacional sobre el "Nuevo Paradigma de la Ciencia de la Educación: la Posibilidad de Ser". Mexicali (México).

MAYOR ZARAGOZA, F. (2000). Un mundo nuevo. Barcelona, UNESCO, Círculo de Lectores.

VILCHES, A. y GIL, D. (2003). Construyamos un futuro sostenible. Diálogos de supervivencia. Madrid. Cambridge University Press.

\section{PÁGINAS WEB}

Ministerio de Educación del Perú: http://www. minedu.gob.pe

Banco Mundial: http://www.bancomundial.org/

Eikasia. Revista de Filosofía, Año II, N ${ }^{\circ} 11$ (julio 2007). Web: http://www.revistadefilosofia. org

El Estado del Futuro 2007. En: http://www.stateofthefuture.org

Instituto Internacional de Planeamiento de la Educación (Buenos Aires). Web: http://www. iipe-buenosaires.org.ar

\section{CUADRO № 1}

\begin{tabular}{|l|l|}
\hline \multicolumn{1}{|c|}{ DE } & \multicolumn{1}{c|}{ A } \\
\hline 1. Del alivio de la pobreza. & Al desarrollo. \\
\hline 2. De la educación como sector. & A la educación como política trans sectorial. \\
\hline 3. Del predominio de los criterios económicos. & A una visión integral de cuestión educativa. \\
\hline 4. De la ayuda internacional. & A una auténtica cooperación internacional. \\
\hline 5. De la escuela. & A la educación. \\
\hline 6. Del derecho a la educación. & Al derecho a la buena educación. \\
\hline 7. Del derecho al acceso. & Al derecho al aprendizaje. \\
\hline 8. Del derecho al aprendizaje. & Al derecho al aprendizaje a lo largo de toda la vida. \\
\hline 9. De la escuela. & A la comunidad de aprendizaje. \\
\hline 10. De la capacitación docente. & A la cuestión docente. \\
\hline 11. De la educación básica como educación escolar. & A la educación básica como educación ciudadana. \\
\hline 12. De adecuarse al cambio. & A incidir sobre el cambio. \\
\hline
\end{tabular}

Fuente: Torres, 2005. 


\section{CUADRO № 2}

Iniciativas y planes internacionales para la educación (1981 - 2017)

\begin{tabular}{|c|c|c|c|c|c|}
\hline 1981-2000 & $1990-2015$ & 1994-2010 & 2000-2015 & 2001-2015 & 2002-2017 \\
\hline $\begin{array}{c}\text { Proyecto principal } \\
\text { de educación para } \\
\text { A. Latina y el Caribe } \\
\text { (regional) }\end{array}$ & $\begin{array}{c}\text { Educación } \\
\text { para todos } \\
\text { (mundial) }\end{array}$ & $\begin{array}{l}\text { Cumbres de } \\
\text { las Américas } \\
\text { (hemisférica) }\end{array}$ & $\begin{array}{l}\text { Objetivos de } \\
\text { desarrollo } \\
\text { del milenio } \\
\text { (mundial) }\end{array}$ & $\begin{array}{c}\text { Cumbres } \\
\text { Ibero } \\
\text { Americanas } \\
\text { (Iberoamérica) }\end{array}$ & $\begin{array}{c}\text { PRELAC } \\
\text { Proyecto Regional de } \\
\text { Educación en A. Latina } \\
\text { y el Caribe (regional) }\end{array}$ \\
\hline UNESCO- OREALC & \begin{tabular}{|} 
UNESCO-UNICEF \\
PNUD-B. MUNDIAL
\end{tabular} & USA-OEA & \begin{tabular}{|c|} 
NNUU-OCDE-FMI \\
B. MUNDIAL
\end{tabular} & $\begin{array}{l}\text { ESPAÑA- } \\
\text { OEI-SGIB }\end{array}$ & UNESCO-OREALC \\
\hline $\begin{array}{l}\text { - } 8 \text { años de educa- } \\
\text { ción general. } \\
\text { - Eliminar el analfa- } \\
\text { betismo. } \\
\text { - Mejorar calidad y } \\
\text { eficiencia. }\end{array}$ & $\begin{array}{l}\text { - Educación básica } \\
\text { de calidad para to- } \\
\text { dos (niños, jóve- } \\
\text { nes, adultos) } \\
\text { - Reducir el analfa- } \\
\text { betismo a la mitad. }\end{array}$ & $\begin{array}{l}\text { - Conclusión univer- } \\
\text { sal educación pri- } \\
\text { maria de calidad. } \\
\text { - Acceso a educación } \\
\text { secundaria de cali- } \\
\text { dad. } \\
\text { - Oportunidades de a- } \\
\text { prendizaje a lo largo } \\
\text { de toda la vida. }\end{array}$ & $\begin{array}{l}\text { - Terminar educa- } \\
\text { ción primaria (4 } \\
\text { años). } \\
\text { - Equidad de gé- } \\
\text { nero en prima- } \\
\text { riay secundaria. }\end{array}$ & $\begin{array}{l}\text { - Ed. inicial. } \\
\text { - Ed. primaria y } \\
\text { básica. } \\
\text { - Universalizar } \\
\text { ed. secundaria. } \\
\text { - Universalizar al- } \\
\text { fabetización y } \\
\text { ed. básica jóve- } \\
\text { nes y adultos. }\end{array}$ & $\begin{array}{l}\text { - Ed. inicial. } \\
\text { - Ed. básica. } \\
\text { - Ed. general, técnica y } \\
\text { profesional. } \\
\text { - Ed. superior. } \\
\text { - Alfabetización. } \\
\text { - Aprendizaje a lo lar- } \\
\text { go de toda la vida. }\end{array}$ \\
\hline
\end{tabular}

Fuente: Torres, 2005. 